\title{
Os efeitos de uma proposta didática de Educação Auditiva na performance musical
}

\author{
The effects of didactic proposal of Hearing Education in musical performance
}

\author{
Mário Cardoso \\ Instituto Politécnico de Bragança, Bragança, Portugal \\ Centro de Investigação em Educação Básica, Bragança, Portugal \\ cardoso@ipb.pt \\ Elsa Morgado \\ Universidade de Trás-os-Montes e Alto Douro, Vila Real, Portugal \\ Centro de Estudos Filosóficos e Humanísticos, Universidade Católica, Portugal \\ levielsa@utad.pt

\section{Levi Silva} \\ Universidade de Trás-os-Montes e Alto Douro, Vila Real, Portugal \\ Centro de Investigação em Ciências e Tecnologias das Artes, Universidade Católica, Portugal \\ levileon@utad.pt
}

Resumo: Um dos desafios do contexto musical educativo contemporâneo é a urgência da construção e desenvolvimento de uma visão pluridisciplinar e multidimensional que possa, por um lado, contribuir para a superação de práticas pedagógicas fragmentadas do contexto e sintaxe musical, e por outro, contextualizar e desenvolver um conjunto de capacidades auditivas, cognitivas e expressivas. Este facto é observado nas práticas pedagógicas das unidades curriculares associadas à construção e desenvolvimento da formação musical dos indivíduos, cujos os paradigmas são pautados pelo desenvolvimento técnico-expressivo, isolamento de elementos fundamentais da sintaxe musical e desfasamento entre a produção contemporânea e seus processos de ensino e aprendizagem. Partindo da pertinência e contemporaneidade que estas práticas pedagógicas levantam no contexto atual foi desenvolvida uma abordagem metodológica de carácter experimental, com a finalidade de verificar se a implementação de um modelo pluridisciplinar e multidimensional de educação auditiva, produz efeitos na melhoria do desempenho e compreensão musical. Esta investigação empírica foi desenvolvida em contexto de Ensino Superior, com recurso a uma linha metodológica experimental, com grupo de controlo com pré-teste e pós-teste. Da análise e interpretação dos resultados emergem indicadores de que o processo de ensino e aprendizagem assente no Modelo Multidimensional de Educação Auditiva, contribui para o desenvolvimento da aprendizagem e reconhecimento de elementos (estrutura rítmica e métrica, organização melódica e harmónica, elementos expressivos e diversidade estilística e instrumental) fundamentais na compreensão da sintaxe musical.

Palavras-chave: educação auditiva; treino auditivo; performance musical.

Abstract: One of the challenges of the contemporary educational musical context is the urgency of building and developing a multidisciplinary and multidimensional vision that can, on the one hand, contribute to the overcoming of fragmented pedagogical practices of the context and musical syntax, and, on the other hand, contextualize and develop a set of auditory, cognitive and expressive abilities. This fact is observed in the pedagogical practices of the curricular units associated to the construction and development of the musical formation of the individuals, whose paradigms are based on the technical-expressive development, isolation of fundamental elements of the musical syntax and lag between contemporary production and its teaching processes and learning. Based on the relevance and contemporaneousness of these pedagogical practices in the current context, an experimental, methodological approach was developed to verify if the implementation of a multidimensional and multidimensional model of auditory education affects performance improvement and 
understanding. This empirical research was developed in a context of Higher Education, using an experimental, methodological line, with a control group with pre-test and post-test. From the analysis and interpretation of the results emerge indicators that the teaching and learning process is based on the Multidimensional Model of Auditory Education, contributes to the development of learning and recognition of elements (rhythmic and metrical structure, melodic and harmonic organization, expressive elements and stylistic and instrumental diversity) fundamental in the understanding of musical syntax.

Keywords: hearing education; ear training; music performance

Submission date: 22 January 2017

Final approval date: 15 July 2018

\section{1 - Introdução}

A condição e a diversidade musical atuais reforçam a ideia da necessidade de abertura, procura e invenção do sentido tradicional do pensamento educativo que impera no universo artístico, em particular no domínio musical. Veja-se o caso da disciplina de Formação Musical, que sendo epígona de objetivos centrados no domínio da escrita e leitura musical (CRUZ, 2013), representa para muitos um local onde a expressão "exercício auditivo", centrado em processos de reprodução diária de "ditados, solfejos e melodias estereotipadas", faz todo o sentido. Este paradigma resulta claramente da importância e da "condição de possibilidade" que a partitura assume na tradição ocidental (VARGAS, 2013). No nosso entendimento, estes processos apresentam pouco valor artístico (BARBOSA, 2009; OTUTUMI, 2008), uma vez que numa tentativa de criação de automatismos de apreciação e compreensão musical são trabalhados unicamente elementos constituintes do discurso musical. O objetivo principal da disciplina é no nosso ponto de vista descurado, já que ficam de parte o desenvolvimento da "memória, audição interior" e toda a "capacidade criativa" (CRUZ, 2013). A compressão do discurso musical na sua totalidade deveria ser princípio estratégico e pensamento "primo" na abordagem pedagógica neste domínio, partindo-se da “... percepção global, para dai perceber as relações desse todo com as suas partes e, nessas partes buscar as estruturas que as sustentam e, por conseguinte, sustentam o todo..." (BERNARDES, 2001, p.77). A este facto devemos acrescentar que a separação da Música do seu contexto coloca de parte aspetos fundamentais para a construção de um julgamento estético como a novidade, a genuinidade e o epigonismo (DAHLHAUS, 1977). 0 (re)pensar destas práticas e abordagens pedagógicas tem sido um fator expresso por diversos autores (CARDOSO, 2015; CRUZ, 2013; BARBOSA, 2009; OTUTUMI, 2008: GREEN, 2008; CASPURRO, 2006; BERNARDES, 2001; RODRIGUES, 1998), apoiados no pensamento, sentimento e visão do corpo discente sobre este domínio específico da Teoria e Formação Musical. Neste particular, CASPURRO (2006) assevera que as dificuldades que têm sido demonstradas pelos discentes na compreensão da Música, bem como a pouca eficácia dos paradigmas de ensino em responder a problemas de realização que se encontram para além do ato performativo, reprodução imitativa de notação ou conhecimento de teoria da música (CASPURRO, 2006), representam indicadores e motivos de reflexão para todo o corpo docente. Para quem se encontra em contacto diário com a realidade educativa, revê nestas palavras a desorientação manifestada pelo corpo discente quando confrontados com práticas como a "improvisação, transposição, composição, compreensão harmónica, leitura à primeira vista" ou mesmo a "execução de ouvido" de uma melodia ou "progressão harmónica" no instrumento musical (CARDOSO, 2015). 
Com o intento de lançar uma perspetiva divergente mas edificante relativamente aos processos de ensino e aprendizagem no domínio musical, a construção e desenvolvimento deste estudo emerge por força de diferentes variáveis a nível epistemológico, metodológico e pedagógico. Assim, na base do seu desenvolvimento estiverem os seguintes fatores:

1. o desejo de mudança e insatisfação em relação às práticas metodológicas, recursos e conceções abraçadas no âmago desta área;

2. a carência de metodologias que permitam explorar e considerar a coexistência dos diferentes domínios da área da Música;

3. a necessidade da existência de uma reconfiguração didática das práticas pedagógicas que possa responder aos desafios e necessidades atuais;

4. a inexistência de uma discussão e reflexão sobre os processos de ensino e aprendizagem na área da Teoria e Formação Musical;

5. a possibilidade de teorizar, construir e implementar uma modelo de ensino e aprendizagem que se possa constituir como uma ferramenta fundamental para o desenvolvimento e compreensão do discurso musical;

6. a possibilidade de promover uma cultura de interdisciplinaridade e pluralidade nas diferentes áreas de investigação da Música;

De todos os sinais e indicadores referenciados resulta a teorização do Modelo Multidimensional de Educação Auditiva (MoMEA), que poderá desempenhar, como processo de (des)construção, um papel relevante no processo de ensino e aprendizagem para a compreensão do discurso musical. Apesar desta tarefa se apresentar árdua, temos a convicção que no domínio do Ensino da Música, este modelo deve representar um procedimento pedagógico, através do qual a aprendizagem musical possa ser entendida como uma realidade que (re)invente e (re)coloque todo em jogo (SISCAR, 2005). Este entendimento será como narrar a "... floresta sem nunca perder de vista as árvores, falar das árvores sem tornar a floresta numa mera abstração..." (SARAMAG0, 2008, p.13).

Dada a consciência da necessidade e importância de refletir sobre esta temática, identificando e introduzindo novos elementos que pensamos essenciais para o melhoramento e reconfiguração didática na área da Música, foram assumidos os seguintes objetivos: (1) conceber e implementar um modelo de ensino e aprendizagem na área da Formação Musical; (2) verificar se a implementação de um conjunto de estratégias experimentais, reunidas no seio do Modelo Multidimensional de Educação Auditiva (MoMEA), produzem efeitos na melhoria do desempenho e compreensão musical; (3) contribuir para a definição e entendimento do conceito de Educação Auditiva.

Considerando todas as questões, objetivos e hipóteses que marcam este estudo, o aprofundamento conceptual e epistemológico do processo de investigação revelou a importância da utilização do design de carácter experimental do grupo de controlo com pré e pós-teste (FREIXO, 2011), opção metodológica que deriva de todo o referencial teórico 
(AZZARA, 2008; GORDON, 2009; AZZARA e GRUNOW, 2006; FIRKE, 1995), onde em termos operacionais este recurso é claramente assumido.

Partindo do objetivo principal deste estudo, centrado na construção e implementação de um conjunto de estratégias/atividades pedagógico-musicais congregadas no Modelo Multidimensional de Educação Auditiva (MoMEA), foi colocada a hipótese de que a eficácia destas estratégias/atividades poderiam ser alvo de verificação na capacidade de Leitura e Execução Musical, mas também no Reconhecimento Auditivo das várias características constituintes do discurso musical. Para cada uma destas componentes e seus indicadores (performativo e cognitivo) foram definidas duas hipóteses: (1) uma hipótese nula, que representa a admissão objetiva de que a ação experimental realizada não provocou alterações nas suas características; (2) uma hipótese alternativa, que consiste numa afirmação relativa às alterações que esperamos que ocorram nas características da amostra definida em função da ação experimental desenvolvida. Todas as hipóteses definidas assumiram-se como elementos orientadores na comparação estatística dos resultados do desempenho musical nas duas componentes definidas (ROVAI, BAKER, PONTON, 2013; FREIXO, 2011; YUNUS, 2010; WEINBERG e ABRAMOWITZ, 2008; TUCKMAN, 2005; BISQUERRA, SARRIERA e MATÍNEZ, 2007; TROCHIM, 2000).

\section{2 - Metodologia}

\section{1 - Participantes}

Participaram neste estudo um total de 42 alunos de ambos os sexos, com idades compreendidas entre 18 e os 49 anos, seletados de acordo com os seguintes critérios: (1) frequência de uma Instituição de Ensino Superior Público ${ }^{1}$ (Universidade e Politécnico); (2) frequência de uma Licenciatura de Música: (3) frequência de uma turma da área da Formação Musical. Os participantes nesta investigação foram divididos com recurso à repartição aleatória (FREIXO, 2011) em dois grupos: Grupo Experimental (N=21) e Grupo de Controlo $(\mathrm{N}=21)$. Esta estratégia de aleatorização permitiu controlar e anular os efeitos de variáveis parasitas e moderadoras em todo este processo de investigação. 0 facto de todos os sujeitos apresentarem a mesma possibilidade de integrar qualquer um dos grupos (Experimental e Controlo), permite o aumento da validade interna e redução do enviesamento.

\section{2 - Instrumentos}

Para este estudo experimental foram construídos e utilizados vários instrumentos de recolha de dados com o objetivo de avaliar a influência no desempenho musical do modelo teorizado - MoMEA. Assim, foram concebidos o Teste de Conhecimento Individual (TCI) e o Teste de Desempenho Musical (TDM). A utilização do Teste de Conhecimento Individual (TCI) teve por base três objetivos fundamentais: (1) obter um perfil individual de cada um dos sujeitos participantes neste estudo de forma a ajustar todo o processo de ensino às características individuais; (2) aferir o nível de conhecimento tonal, rítmico e teórico; (3) verificar a existência de relações com os testes construídos e aplicados numa fase posterior da

${ }^{1}$ As Instituições de Ensino Superior selecionadas para este estudo pertencem aos subsistemas universitário e politécnico. 
investigação. Para que se possa cumprir com os objetivos enunciados, o Teste de Conhecimento Individual (TCI), reúne um conjunto de provas específicas (Prova Tonal, Prova Rítmica, Prova Teórica), constituídas por questões sobre aspetos fundamentais da dimensão Tonal (T), Rítmica (R) e Teórica (Te). Em termos operacionais, este teste (TCI) baseia-se no reconhecimento, com recurso à audição de música gravada em áudio e registo escrito, de aspetos fundamentais do discurso musical. A anotação das respostas referentes a cada um destas provas foi realizada em folha própria, construída pelo investigador, tendo sido alvo numa fase posterior, a uma catalogação e análise dos seus resultados. Na base da construção deste Teste de Conhecimento Individual, estiveram critérios de ordem musical (Tonal, Rítmica, Teórica), cognitivos (Conhecimento, Compreensão, Aplicação, Análise) e técnicos (Apresentação, Avaliação e Duração). Para a correção do TCI foram criadas rating scales de critério numérico (GORDON, 2002; AZZARA, 1993), com cotações diferenciadas para cada uma das dimensões em estudo. Para o registo das classificações obtidas pelos participantes deste estudo empírico foram utilizadas grelhas individuais de correção.

O Teste de Desempenho Musical (TDM), foi construído e desenhado para ser utilizado durante todo o processo de investigação com a finalidade de avaliar, de forma individual, o nível de desempenho dos participantes antes e depois de serem submetidos à instrução. Assim, foram construídos dois Testes de Desempenho Musical (TDM1 e TDM2), subdivididos em duas dimensões: (1) Leitura e Escrita Musical (TLEM); (2) Reconhecimento Auditivo (TRA). 0 primeiro teste (TDM1) cumpre a função de diagnóstico do desempenho musical e o segundo (TDM2) de aferição dos resultados desenvolvidos durante o processo de instrução do MoMEA. As duas dimensões que constituem o TDM, apresentam estruturas e conteúdos diferenciados. No que concerne ao Teste de Leitura e Execução Musical, este é constituído por três provas (Prova Tonal, Prova Rítmica e Prova de Leitura), com exercícios que foram interpretados/executados por cada um dos intervenientes da investigação. Toda a administração desta componente do TLEM foi realizada de forma individual, tendo sido realizado o registo áudio de todas as interpretações/respostas dos participantes.

O Teste de Reconhecimento Auditiva (TRA) foi construído e desenhado para ser utilizado durante todo o processo de investigação, com objetivo de avaliar, de forma individual, antes e depois do período de instrução, o nível de compreensão e reconhecimento auditivo das diferentes categorias presentes nas Atividades de Análise Auditiva: Estrutura Métrica e Rítmica (EMR), Organização Melódica Harmónica (OMH), Elementos Expressivos (EE) e Diversidade Estilística e Instrumental (DEI). Em relação ao seu teor e constituição, este apresenta-se estruturado de acordo com os conteúdos previstos e desenvolvidos pelos sujeitos que integraram esta investigação, formado por vinte questões subdivididas pelas categorias já referidas. Este TRA baseia-se na audição de fragmentos musicais (gravados em formato áudio), sobre os quais todos os sujeitos desta investigação devem responder a um conjunto de questões sobre aspetos determinantes para a análise da(s) obra(s) apresentada(s). Apesar deste instrumento de recolha de dados obedecer a alguns critérios de carácter escrito, este trata-se exclusivamente de um teste auditivo. Para que todos os sujeitos deste estudo empírico se possam familiarizar com o objetivo e a natureza dos diferentes testes foi realizada uma sessão preparatória. Para a correção do TDM, foram criadas rating scales para ambas as componentes (Leitura e Execução Musical e Reconhecimento Auditivo). No caso da Leitura e Execução Musical foram utilizadas rating scales de critérios contínuos e aditivos (GORDON, 2002; AZZARA, 1993), para avaliar as dimensões Tonal (T), Rítmica (R) e 
Leitura (L). Em relação à correção da componente Reconhecimento Auditivo, optou-se pela criação e utilização de rating scales de cinco critérios aditivos (GORDON, 2002; AZZARA, 1993), para a avaliação das seguintes dimensões: Estrutura Métrica e Rítmica (EMR), Organização Melódica Harmónica (OMH), Elementos Expressivos (EE) e Diversidade Estilística e Instrumental (DEI). No que concerne ao registo das classificações referente às duas componentes (Leitura e Execução Musical e Reconhecimento Auditivo) que constituem estes TDM, foram elaboradas fichas de avaliação para cada um dos sujeitos participantes neste estudo empírico.

\section{3 - Procedimentos}

Toda a investigação é composta por várias fases e etapas, onde a importância e a interatividade entre elas desempenham um fator fulcral no atingir dos objetivos iniciais de qualquer estudo empírico (FREIXO, 2011; TUCKMAN, 2005; FORTIN, 1999). Deste modo, o presente estudo encontra-se dividido em quatro fases distintas.

A primeira fase do estudo empírico combina a definição, constituição e divisão da amostra em dois grupos distintos (Grupo Experimental e Grupo de Controlo), com a recolha de dados iniciais que caracterizam os níveis de conhecimento individual dos participantes, a nível da dimensão Tonal, Rítmica e Teórica (TCI), e níveis de desempenho musical (TDM), nas componentes da Leitura/Execução musical (TLEM1) e Reconhecimento Auditivo (TRA1). Estes testes, para além de servirem de referencial para o restante percurso empírico permitiram que todos os sujeitos deste estudo se familiarizassem com todo o processo.

A segunda fase do estudo empírico corresponde ao período de instrução musical, sendo aplicado ao Grupo Experimental os procedimentos e orientações metodológicas previstas no modelo MoMEA, e ao Grupo de Controlo as linhas dominantes de ensino e aprendizagem identificadas durante o estudo exploratório desenvolvido em processos investigativos anteriores (CARDOSO, 2015). Esta instrução decorreu entre setembro de 2013 e junho de 2014, através de uma sessão semanal, com a duração de 30 minutos para cada um dos grupos (Experimental e Controlo), numa totalidade de 40 sessões. De forma a manter a confidencialidade e exequibilidade de todo o processo, foi atribuído a cada um dos participantes uma identificação numérica. Para a organização de cada uma das sessões de trabalho do MoMEA, foi utilizada a linha conceptual de GORDON (2000), de Atividades Sequenciais e Atividades de Sala de Aula, adotando-se para a organização de cada uma das sessões um critério de Todo-Parte-Todo (TAGGART, BOLTON, REYNOLDS, VALERIO, GORDON, 2006; LANGE, 2005; KNOWLES, HOLTON e SWANSON, 2005; BOLTON, TAGGART, REYNOLDS, VALERIO, GORDON, 2001; GORDON; 2000; BLUESTINE, 1995). Em relação à instrução e estruturação de todo o procedimento metodológico, cada uma das unidades didáticas desenvolvidas, apresentou-se estruturada de acordo com três categorias diferenciadas: (1) Atividades de Repertório; (2) Atividades de Análise Auditiva; (3) Atividade de Contexto. A interligação e complementaridade entre estas categorias representa um fator fundamental na coesão deste modelo (MoMEA).

As Atividades de Repertório representam para o MoMEA, um elemento fundamental como princípio para o desenvolvimento e aprendizagem do discurso musical. Promover uma aprendizagem através do ouvido de diversos estilos, tonalidades e métricas, revela-se um grande desafio e fator de importância no processo de ensino e aprendizagem (GORDON, 2000; 
AZZARA, 2008). Tendo em conta a diversidade dos contextos pedagógicos, o repertório utilizado nestas atividades apresenta uma variedade de estilos, géneros e épocas. Este facto, para além de possibilitar uma contextualização histórica e estilística de todo o universo musical, contribui para o desenvolvimento de toda a sensibilidade auditiva, fundamental na construção de uma escuta consciente e compreensiva do discurso musical. Estas atividades ocupam a fase inicial de cada sessão de trabalho, tendo uma duração aproximada de 5 minutos.

Partindo da premissa que todos os principais elementos que configuram o universo musical são passíveis de reconhecimento auditivo, as Atividades de Análise Auditiva pretendem desenvolver competências de audição musical a nível da identificação, categorização e análise dos diferentes atributos presentes no discurso musical. A diferença em relação às restantes atividades, advém do facto de estas representarem no processo global de ensino a função da aprendizagem das categorias (partes) dos conteúdos essenciais. Em todo o processo de ensino e aprendizagem, estas atividades apresentam uma duração aproximada de 15 minutos, contemplando um critério direcional de Todo-Parte-Todo.

As Atividades de Contexto resultam da relação entre os conteúdos essenciais e conteúdos transversais definidos, constituindo-se como atividades complementares a nível melódico, rítmico e expressivo, em contexto de expressão e performance musical. Tendo aproximadamente uma duração de 10 minutos, estas atividades apresentam a função de aprendizagem do Todo no processo sequencial de sala de aula. Em aspetos relacionados com a aprendizagem de conteúdos este tipo de atividades integram as três dimensões definidas (Ritmo, Melodia e Harmonia).

Após a realização do período de instrução, procedeu-se à avaliação individual dos níveis de desempenho musical (TLEM 2 e TRA 2), nas duas componentes definidas para este estudo (Leitura/Execução Musical e Reconhecimento Auditivo). O procedimento utilizado para a avaliação individual seguiu a mesma linha metodológica dos testes iniciais (TCI).

A última fase corresponde ao tratamento e análise dos dados. Assim, os dados relativos aos diversos testes realizados foram tratados e analisados de uma forma quantitativa, com recurso à estatística descritiva e inferencial, com o intuito de conduzir os resultados obtidos para a validação e/ou refutação das hipóteses iniciais colocadas para este estudo empírico. Na Figura 1 pode ser observada toda a esquematização do procedimento adotado para o estudo empírico de carácter experimental realizado. 


\section{Procedimento}

\begin{tabular}{|c|}
\hline Amostra \\
\hline Perfil de Conhecimento Musical \\
\hline
\end{tabular}

40 semanas de instrução (duração de 30 minutos cada)

\begin{tabular}{|c|c|}
\hline Grupo Experimental & Grupo de Controlo \\
Ensino Musical com a instrução do MoMEA & Ensino Musical \\
\hline
\end{tabular}

Critérios de medida:

Escalas de Classificação

Desempenho Musical

Figura 1: Procedimento do Estudo.

\section{3 - Método e Tratamento de Dados}

Respeitando o carácter quantitativo e experimental desta investigação (FREIXO, 2011; FORTIN, 1999), foi construída uma base de dados, com o objetivo de analisar os resultados apurados e responder/testar as hipóteses definidas. Todo este procedimento envolveu três etapas: (1) Preparação de Dados; (2) Estatística Descritiva; (3) Estatística Inferencial (TROCHIM, 2000). Assim, num primeiro momento foi realizada a verificação, organização e registo dos dados relativos aos diferentes testes utilizados no estudo empírico. Após a preparação destes dados, os procedimentos estatísticos que se seguiram passaram pela utilização da Estatística Descritiva e Estatística Inferencial. A utilização da Estatística Descritiva (Frequências, Médias e Desvio Padrão) teve por objetivo a descrição e sintetização de todos os dados previamente recolhidos, verificados e organizados. Por outro lado, através da Estatística Inferencial (Teste de Kolmogorov-Smirnov, Teste de Levene e Teste $t$-Student), foram testadas todas as hipóteses enunciadas (ROVAI, BAKER e PONTON, 2013; YUNUS, 2010; WEINBERG e ABRAMOWITZ, 2008; TUCKMAN, 2005; BISQUERRA, SARRIERA e MATÍNEZ, 2007; TROCHIM, 2000). Neste particular, TUCKMAN $(2005$, p.369) refere que através da “... testagem estatística, um investigador pode comparar grupos de dados, de modo a determinar qual a probabilidade da diferença entre eles se basear no acaso, proporcionando assim as provas para ajuizar da validade de uma hipótese ou inferência...". Para a verificação da consistência interna foram utilizados o método de Alpha de Cronbach e a fórmula de 
Spearman-Brown (TUCKMAN, 2005; BISQUERRA, SARRIERA e MATÍNEZ, 2007; TROCHIM, 2000). No caso da validade de constructo foi utilizada uma análise fatorial (amostra total), com recurso ao método de componentes principais e rotação de varimax. Para a verificação das condições de aplicabilidade destes testes foram utilizados os testes de Kaiser-Meyer-Olkin (KMO) e Bartlett (ROVAI, BAKER e PONTON, 2013; YUNUS, 2010; WEINBERG e ABRAMOWITZ, 2008). A base de dados foi construída com o recurso ao programa de análise estatística Statistical Package for the Social Sciences (SPSS) versão 21.

\section{4 - Resultados}

Através dos dados estatísticos presentes na Figura 2, observamos que grande parte dos indicadores performativos atingem o nível 1 da escala de classificação, à exceção do Percutir e Verbalizar (nível 2). No que respeita aos valores máximos podemos perceber que, de uma forma geral, os valores ascendem para os níveis 4 e 5 (Entoar, Percutir/Fonetizar e Percutir e Verbalizar), sendo atingido o nível 2 para o Solfejo por Relatividade.

\begin{tabular}{ccccc}
\hline & Mínimo & Máximo & Média & Desvio Padrão \\
\hline E (à capella) & 1,0 & 4,0 & 2,80 & 0,98 \\
E (com acomp.) & 1,0 & 4,0 & 2,66 & 0,91 \\
P/F & 1,0 & 5,0 & 2,90 & 1,17 \\
PV & 2,0 & 5,0 & 3,04 & 0,92 \\
SR & 1,0 & 2,0 & 1,14 & 0,35 \\
\hline
\end{tabular}

Figura 2: Resultados Estatísticos descritivos para a Leitura e Execução Musical do Grupo Experimental (E significa entoar, P/F significa percutir/fonetizar, PV significa percutir e verbalizar, e SR significa Solfejo por Relatividade).

A Figura 2 aponta ainda para uma média elevada para o Percutir e Verbalizar $(M=3,04$; e $\mathrm{DP}=0,92)$, e para o Percutir /Fonetizar (M=2,90; e $\mathrm{DP}=1,17)$. A média mais baixa foi verificada no Solfejo por Relatividade (M=1,14; e $\mathrm{DP}=0,35)$. Relativamente ao Grupo de Controlo (Figura 3), os valores mínimos obtidos para a totalidade dos seus elementos situaram-se no nível 1. No que respeita aos valores máximos podemos destacar o Percutir/Fonetizar (nível 5), o Entoar com acompanhamento (nível 4) e o Percutir e Verbalizar (nível 4).

\begin{tabular}{ccccc}
\hline & Mínimo & Máximo & Média & Desvio Padrão \\
\hline E (à capella) & 1,0 & 3,0 & 1,95 & 0,86 \\
E (com acomp) & 1,0 & 4,0 & 1,80 & 1,12 \\
P/F & 1,0 & 5,0 & 2,52 & 1,53 \\
PV & 1,0 & 4,0 & 1,80 & 0,98 \\
SR & 1,0 & 2,0 & 1,57 & 0,50 \\
\hline
\end{tabular}

Figura 3: Resultados Estatísticos descritivos para a Leitura e Execução Musical do Grupo de Controlo (E significa entoar, P/F significa percutir/fonetizar, PV significa percutir e verbalizar, e SR significa Solfejo por Relatividade).

Em relação aos valores das médias alcançados pelo Grupo de Controlo (Figura 2), foram obtidos, comparativamente ao Grupo Experimental, valores inferiores em toda a sua amplitude. Estes resultados constituem um fortíssimo indicador de que o processo de 
instrução (MoMEA) possa ter contribuído para esta diferenciação. Assim, a média mais elevada foi verificada no Percutir/Fonetizar $(M=2,52$; e $D P=1,53)$, sendo atingida a média mais baixa para o Solfejo por Relatividade $(\mathrm{M}=1,57$; e $\mathrm{DP}=0,50)$. De uma forma geral os valores das médias, com a exceção do Percutir/Fonetizar, tiveram valores abaixo do nível 2. Estando os valores aritméticos apresentados associados a escalas de classificação (rating scale), importa perceber em que níveis esses valores se situam. Será a partir desses mesmos valores que podemos perceber os ganhos significativos em relação ao indicador performativo. Neste sentido, são apresentados na Figura 3, as frequências dos níveis da escala de classificação referentes ao Grupo Experimental.

Pela observação e leitura da Figura 4, podemos perceber que para todos os elementos constituintes do indicador performativo, o grau de frequência (GF) mais elevado, refere-se ao nível 3, situando-se entre os 23,8\% para a Entoação com acompanhamento, e os 52,4\% correspondente ao Percutir/Fonetizar. De salientar, que nos indicadores Percutir/Fonetizar $(\mathrm{GF}=3)$ e Percutir e Verbalizar ( $\mathrm{GF}=2$ ), foram registados os valores máximos de nível 5 da escala de classificação. Estes valores, permitem evidenciar que os participantes do presente estudo, apresentam na sua maioria, características de inegável relevância a nível do domínio da coordenação psico-motora, executando todos os padrões em todas as funções de uma forma correta. No caso do indicador Entoação, podemos observar a existência de uma diferenciação entre as condições de à capella e com acompanhamento, existindo no caso desta última, uma maior predominância a nível do conservar o sentido de tonalidade. Este facto permite estabelecer uma fortíssima relação com o acompanhamento harmónico realizado. De destacar os valores bastante baixos atingidos no Solfejo por Relatividade - grau de frequência de $18(85,7 \%)$ obtidos no nível 1 da escala de classificação. Contudo, estes valores já eram de alguma forma esperados, devido às particularidades e carácter auditivo/oral intrínseco ao nosso processo de instrução. De forma análoga aos procedimentos realizados para o Grupo Experimental, as distribuições e frequências referentes ao indicador performativo do Grupo de Controlo foram compactadas e podem ser observadas na Figura 5.

\begin{tabular}{|c|c|c|c|c|c|c|}
\hline Frequências nas 5 provas constituintes do TLEM & 1 & 2 & 3 & 4 & 5 & Totais \\
\hline \multirow{2}{*}{ P0T1 } & 2 & 6 & 7 & 6 & - & 21 \\
\hline & $9,5 \%$ & $28,6 \%$ & $33,3 \%$ & $28,6 \%$ & - & $100 \%$ \\
\hline \multirow[t]{2}{*}{ РOT2 } & 1 & 10 & 5 & 5 & - & 21 \\
\hline & $4,8 \%$ & $47,6 \%$ & $23,8 \%$ & $23,5 \%$ & - & $100 \%$ \\
\hline \multirow[t]{2}{*}{ POR3 } & 3 & 3 & 11 & 1 & 3 & 21 \\
\hline & $14,3 \%$ & $14,3 \%$ & $52,4 \%$ & $4,8 \%$ & $14,3 \%$ & $100 \%$ \\
\hline \multirow[t]{2}{*}{ POR4 } & - & 6 & 10 & 3 & 2 & 21 \\
\hline & - & $28,6 \%$ & $47,6 \%$ & $14,3 \%$ & $9,5 \%$ & $100 \%$ \\
\hline \multirow[t]{2}{*}{ SR } & 18 & 3 & - & - & - & 21 \\
\hline & 85,7 & 14,3 & - & - & - & $100 \%$ \\
\hline
\end{tabular}

Figura 4: Distribuição e Frequência do Resultados da Leitura e Execução Musical por pontuação do Grupo Experimental (POT significa prova oral tonal, POR significa prova oral rítmica, e SR significa solfejo por relatividade). 
CARDOSO, M.; MORGADO, E.; SILVA, L. (2018) Os efeitos de uma proposta didática de Educação Auditiva no desempenho musical. Per Musi. Belo Horioznte: UFMG. p.1-18.

\begin{tabular}{lcccccc}
\hline Frequências nas 5 provas constituintes do TLEM & 1 & 2 & 3 & 4 & 5 & Totais \\
\hline POT1 & 8 & 6 & 7 & 6 & - & 21 \\
& $38,1 \%$ & $28,6 \%$ & $33,3 \%$ & $28,6 \%$ & - & $100 \%$ \\
POT2 & 12 & 4 & 2 & 3 & - & 21 \\
& $57,1 \%$ & $19,0 \%$ & $9,5 \%$ & $14,3 \%$ & - & $100 \%$ \\
POR3 & 7 & 6 & 2 & 2 & 4 & 21 \\
POR4 & $33,3 \%$ & $28,6 \%$ & $9,5 \%$ & $9,5 \%$ & $19,0 \%$ & $100 \%$ \\
SR & 10 & 7 & 2 & 2 & - & 21 \\
& $47,6 \%$ & $33,3 \%$ & $9,5 \%$ & $9,5 \%$ & - & $100 \%$ \\
& 9 & 12 & - & - & - & 21 \\
\hline
\end{tabular}

Figura 5: Distribuição e Frequência dos Resultados Estatísticos Descritivos da Leitura e Execução Musical por pontuação do Grupo de Controlo (POT significa prova oral tonal, POR significa prova oral rítmica, e SR significa solfejo por relatividade).

Da análise dos dados expostos na Figura 5, ressalta que o nível 1 é de uma forma geral, o elemento de maior predominância, situando-se entre os $33,3 \%$ relativos ao Percutir/Fonetizar e os 57,1\% do Entoar com acompanhamento. De uma forma geral, os valores obtidos pelo Grupo de Controlo apresentam-se inferiores em todas as dimensões, quando comparados com os valores atingidos pelo Grupo Experimental. Todos estes elementos representam indicadores de relevo da não influência da variável independente definida, o que permite ir consolidando a hipótese que pauta esta pesquisa. No que diz respeito ao Solfejo por Relatividade, os valores obtidos apresentam indicadores superiores em relação aos valores do Grupo Experimental, apontando claramente toda a linha metodológica de valorização da associação simbólica, como objetivo primário, inerente ao modelo de ensino e aprendizagem presente nas práticas pedagógicas existentes no universo musical atual. Ao contrário do que foi apurado no Grupo Experimental, o nível 5 unicamente foi verificado no indicador Percutir/Fonetizar, apresentando um grau de frequência de 4 $(19,0 \%)$. A Figura 6 apresenta os resultados estatísticos obtidos pelo Grupo Experimental (pós-teste), relativos ao indicador cognitivo na sua componente Reconhecimento Auditivo.

\begin{tabular}{ccccc}
\hline & Mínimo & Máximo & Média & Desvio Padrão \\
\hline ERM & 2,0 & 4,0 & 3,19 & 0,60 \\
OMH & 2,0 & 5,0 & 3,23 & 0,70 \\
EE & 2,0 & 4,0 & 3,33 & 0,57 \\
DEI & 3,0 & 5,0 & 3,61 & 0,58 \\
\hline
\end{tabular}

Figura 6: Resultados Descritivos para o Reconhecimento Auditivo do Grupo Experimental (ERM significa estrutura rítmica e métrica, OMH significa organização melódica e harmónica, EE significa elementos expressivos, e DEI significa diversidade estilística e instrumental

Como podemos observar, os valores mínimos situaram-se no nível 2 para as componentes de reconhecimento da Estrutura Rítmica e Métrica, Organização Melódica e Harmónica e Elementos Expressivos, sendo de nível 3 para a componente Diversidade Estilística e Instrumental. Em relação aos seus máximos, todos os valores crescem para os níveis 5 (Organização Melódica e Harmónica e Diversidade Estilística e Instrumental) e nível 4 (Estrutura Rítmica e Métrica e Elementos Expressivos). Atendendo ainda aos dados expostos, 
verifica-se que de uma forma geral, a média centrou-se no nível 3, apresentando o seu valor máximo na componente Diversidade Estilística e Instrumental $(M=3,61$; e $D P=0,58)$, sendo o valor mínimo obtido pela componente Estrutura Rítmica e Métrica $(M=3,19$; e $\mathrm{DP}=0,60)$.

Relativamente aos resultados estatísticos alcançados pelo Grupo de Controlo, podemos constatar, que de uma forma geral, os resultados apresentam-se ligeiramente inferiores quando comparados ao Grupo Experimental. Como podemos notar na Figura 7, o valor mínimo situou-se no nível zero para todas as componentes, sendo o valor máximo de nível 5 para a componente Diversidade Estilística e Instrumental. Comparativamente ao Grupo Experimental, os valores das médias, revelam uma ligeira inferioridade em toda a sua amplitude, sendo o valor máximo verificado na componente Diversidade Estilística e Instrumental $(M=3,23$; e $\mathrm{DP}=1,04)$ e o valor mínimo na componente Estrutura Rítmica e Métrica (M=2,52; e DP=0,74) e Organização Melódica e Harmónica (M=2,52; e DP=0,98). Pela observação dos elementos fornecidos pela Figura 8, ressalta desde logo, a elevada predominância dos níveis 3 e 4 em todas as componentes do indicador cognitivo. 0 nível 3 apresenta um grau de frequência que varia de $9(42,9 \%)$, referente à componente Diversidade Estilística e Instrumental e 13 (61,9\%) para as componentes de Estrutura Rítmica e Métrica e Organização Melódica e Harmónica. No caso do nível 4, este apresenta uma variação do grau de frequência que vai deste 5 (23,8\%) da componente Organização Melódica e Harmónica e $11(52,9)$ na componente Diversidade Estilística e Instrumental. De realçar que existiram duas componentes onde foram atingidos o nível máximo (nível 5) da escala de classificação e ainda a não existência de qualquer grau de frequência no nível 1.

\begin{tabular}{ccccc}
\hline & Mínimo & Máximo & Média & Desvio Padrão \\
\hline ERM & 0,0 & 3,0 & 2,52 & 0,74 \\
OMH & 0,0 & 4,0 & 2,52 & 0,98 \\
EE & 0,0 & 4,0 & 2,95 & 0,92 \\
DEI & 0,0 & 5,0 & 3,23 & 1,04 \\
\hline
\end{tabular}

Figura 7: Resultados Estatísticos Descritivos para o reconhecimento Auditivo do Grupo de Controlo (ERM significa estrutura rítmica e métrica, OMH significa organização melódica e harmónica, EE significa elementos expressivos, e DEI significa diversidade estilística e instrumental

\begin{tabular}{lccccccc}
\hline Frequências nas 5 provas constituintes do TRA & $\mathbf{0}$ & $\mathbf{1}$ & $\mathbf{2}$ & $\mathbf{3}$ & $\mathbf{4}$ & $\mathbf{5}$ & Totais \\
\hline ERM & - & - & 2 & 13 & 6 & - & 21 \\
& - & - & $9,5 \%$ & $61,9 \%$ & $28,6 \%$ & - & $100 \%$ \\
OMH & - & - & 2 & 13 & 5 & 1 & 21 \\
& - & - & $9,5 \%$ & $61,9 \%$ & $23,8 \%$ & $4,8 \%$ & $100 \%$ \\
EE & - & - & 1 & 12 & 8 & - & 21 \\
& - & - & $4,8 \%$ & $57,1 \%$ & - & - & $100 \%$ \\
DEI & - & - & - & 9 & 11 & 1 & 21 \\
& - & - & - & $42,9 \%$ & $52,9 \%$ & $4,8 \%$ & $100 \%$ \\
\hline
\end{tabular}

Figura 8: Distribuição e Frequência dos Resultados do Reconhecimento Auditivo por pontuação do Grupo Experimental (ERM significa estrutura rítmica e métrica, OMH significa organização melódica e harmónica, EE significa elementos expressivos, e DEI significa diversidade estilística e instrumental 
No caso do Grupo de Controlo os resultados (Figura 9) apontam para uma predominância do nível 3 da escala de classificação para todas as componentes do indicador cognitivo, apresentando valores bastante semelhantes, com uma única exceção na componente Organização Melódica e Harmónica, do grau de frequência verificados no Grupo Experimental. Apesar desta similitude de resultados entre os dois grupos participantes no estudo referentes ao nível 3, existe uma diferenciação em níveis superiores (nível 4), onde é clara a vantagem do Grupo Experimental. Esta distinção pode ser ainda observada e verificada nos níveis inferiores onde, ao contrário do Grupo Experimental, existem graus de frequência nos níveis 1 e 0.

\begin{tabular}{lccccccc}
\hline Frequências nas 5 provas constituintes do TRA & $\mathbf{0}$ & $\mathbf{1}$ & $\mathbf{2}$ & $\mathbf{3}$ & $\mathbf{4}$ & $\mathbf{5}$ & Totais \\
\hline ERM & 1 & - & 7 & 13 & 0 & - & 21 \\
& $4,8 \%$ & - & $33,3 \%$ & $61,9 \%$ & $0,0 \%$ & - & $100 \%$ \\
OMH & 1 & 1 & 8 & 8 & 3 & - & 21 \\
& $4,8 \%$ & $4,8 \%$ & $38,1 \%$ & $38,1 \%$ & $14,3 \%$ & - & $100 \%$ \\
EE & 1 & - & 3 & 12 & 5 & - & 21 \\
& $4,8 \%$ & - & $14,3 \%$ & $57,1 \%$ & $23,8 \%$ & - & $100 \%$ \\
DEI & 1 & - & 2 & 9 & 8 & 1 & 21 \\
& $4,8 \%$ & - & $9,5 \%$ & $42,9 \%$ & $38,1 \%$ & $4,8 \%$ & $100 \%$ \\
\hline
\end{tabular}

Figura 9: Distribuição e Frequência dos Resultados do Reconhecimento Auditivo por pontuação do Grupo de Controlo (ERM significa estrutura rítmica e métrica, OMH significa organização melódica e harmónica, EE significa elementos expressivos, e DEI significa diversidade estilística e instrumental

Em suma, considerando os testes e procedimentos estatísticos realizados (Figura 10 e 11), podemos afirmar que:

1. todas as variáveis referentes ao indicador cognitivo apresentam normalidade de distribuição e igualdade de variâncias;

2. os testes estatísticos realizados permitem admitir que existem diferenças significativas entre os resultados de desempenho musical, a nível do reconhecimento auditivo, entre o grupo experimental e grupo de controlo relativamente às variáveis Reconhecer a Estrutura Rítmica e Métrica e Reconhecer a Organização Melódica e Harmónica;

3. os testes estatísticos realizados permitem admitir que não existem diferenças significativas entre os resultados de desempenho musical, a nível do reconhecimento auditivo, entre o grupo experimental e grupo de controlo relativamente às variáveis Reconhecer Elementos Expressivos e Reconhecer a Diversidade Estilística e Instrumental;

4. os sujeitos que utilizam estratégias de ensino e aprendizagem (MoMEA) apresentam resultados mais elevados no reconhecimento auditivo da Estrutura Rítmica e Métrica, Organização Melódica e Harmónica, Elementos Expressivos e Diversidade Estilística e Instrumental;

5. a aplicação do Teste de Kolmogorov-Smirnov, apontou para a rejeição da hipótese nula que a distribuição das pontuações é a mesma entre as categorias de grupo para as variáveis Entoar um Texto Musical (condição acompanhamento), Percutir e Verbalizar e Solfejo. 
6. a aplicação do Teste de Levene revelou que as variáveis Entoar um Texto Musical e Percutir e Verbalizar apresentam variâncias homogéneas, tendo-se verificado uma não homogeneidade de variância para as restantes variáveis.

7. os testes estatísticos realizados, permitem admitir que existem diferenças significativas entre os resultados de desempenho musical, a nível da leitura e execução musical, entre o grupo experimental e grupo de controlo relativamente às variáveis Entoar um Texto Musical (condição de à capella e acompanhamento), Percutir e Verbalizar e Solfejo. De salientar que nestas variáveis, com exceção da Entoação de Texto Musical (condição à capella), não podemos aceitar os resultados, pelo motivo de não estarem garantidas as condições de aplicabilidade do teste paramétrico t-Student.

8. os testes estatísticos realizados permitem verificar que não existem diferenças significativas entre os resultados de desempenho musical, a nível leitura e execução musical, entre o grupo experimental e grupo de controlo relativamente à variável Percutir/Fonetizar.

\begin{tabular}{|c|c|c|c|c|c|c|c|c|c|}
\hline Indicador & & Grupo & M & DP & $\begin{array}{l}\text { K-S } \\
\text { Sig. }\end{array}$ & $\begin{array}{l}\text { TL } \\
\text { Sig. }\end{array}$ & $\mathrm{t}$ & $\mathrm{df}$ & Sig \\
\hline \multirow{4}{*}{$\begin{array}{l}\text { Indicador } \\
\text { Cognitivo }\end{array}$} & EMR & $\begin{array}{l}\text { Experimental } \\
\text { Controlo }\end{array}$ & $\begin{array}{l}3,190 \\
2,524\end{array}$ & $\begin{array}{l}0,601 \\
0,749\end{array}$ & 0,358 & 0,320 & 3,17 & 40 & $0,003^{*}$ \\
\hline & $\mathrm{OMH}$ & $\begin{array}{l}\text { Experimental } \\
\text { Controlo }\end{array}$ & $\begin{array}{l}3,238 \\
2,524\end{array}$ & $\begin{array}{l}0,700 \\
0,980\end{array}$ & 0,095 & 0,111 & 2,71 & 40 & $0,010^{*}$ \\
\hline & $\mathrm{EE}$ & $\begin{array}{l}\text { Experimental } \\
\text { Controlo }\end{array}$ & $\begin{array}{l}3,333 \\
2,952\end{array}$ & $\begin{array}{l}0,577 \\
0,920\end{array}$ & 0,983 & 0,788 & 1,60 & 40 & 0,116 \\
\hline & DEI & $\begin{array}{l}\text { Experimental } \\
\text { Controlo }\end{array}$ & $\begin{array}{l}3,619 \\
3,238\end{array}$ & $\begin{array}{l}0,589 \\
1,044\end{array}$ & 0,983 & 0,188 & 1,45 & 40 & 0,153 \\
\hline
\end{tabular}

Figura 10: Resultados estatísticos do indicador cognitivo (M significa média, DP significa desvio de padrão, K-S significa Kolmogorov-Smirnov, TL significa teste de Levene, $\mathrm{t}$ significa $t$-Student, df significa graus de liberdade, e Sig significa nível de significância). ${ }^{*} p<.05$

\begin{tabular}{|c|c|c|c|c|c|c|c|c|c|}
\hline Indicador & & Grupo & M & DP & $\begin{array}{l}K-S \\
\text { Sig. }\end{array}$ & $\begin{array}{c}T L \\
\text { Sig. }\end{array}$ & $t$ & $\mathrm{df}$ & Sig. \\
\hline \multirow{5}{*}{$\begin{array}{l}\text { Indicador } \\
\text { Performativo }\end{array}$} & $\begin{array}{c}\mathrm{E} \\
\text { (à capella) }\end{array}$ & $\begin{array}{l}\text { Experimental } \\
\text { Controlo }\end{array}$ & $\begin{array}{l}2,809 \\
1,952\end{array}$ & $\begin{array}{l}0,980 \\
0,864\end{array}$ & 0,358 & 0,589 & 3,00 & 40 & $0,005^{*}$ \\
\hline & $\underset{\text { (acompl }}{\mathrm{E}}$ & $\begin{array}{l}\text { Experimental } \\
\text { Controlo }\end{array}$ & $\begin{array}{l}2,666 \\
1,809\end{array}$ & $\begin{array}{l}0,912 \\
1,123\end{array}$ & 0,006 & 0,415 & 2,71 & 40 & $0,010^{*}$ \\
\hline & $\mathrm{P} / \mathrm{F}$ & $\begin{array}{l}\text { Experimental } \\
\text { Controlo }\end{array}$ & $\begin{array}{l}2,904 \\
2,523\end{array}$ & $\begin{array}{l}1,179 \\
1,536\end{array}$ & 0,194 & 0,043 & 0,90 & 40 & 0,373 \\
\hline & PV & $\begin{array}{c}\text { Experimental } \\
\text { Controlo }\end{array}$ & $\begin{array}{l}3,047 \\
1,809\end{array}$ & $\begin{array}{l}0,920 \\
0,980\end{array}$ & 0,006 & 0,505 & 4,21 & 40 & $0,000^{*}$ \\
\hline & $S$ & $\begin{array}{c}\text { Experimental } \\
\text { Controlo }\end{array}$ & $\begin{array}{l}1,142 \\
1,571\end{array}$ & $\begin{array}{l}0,358 \\
0,507\end{array}$ & 0,042 & 0,000 & $\begin{array}{c}- \\
3,16\end{array}$ & 40 & $0,003^{*}$ \\
\hline
\end{tabular}

Figura 11: Resultados estatísticos do indicador performativo (M significa média, DP significa desvio de padrão, K-S significa Kolmogorov-Smirnov, TL significa teste de Levene, t significa $t$-Student, df significa graus de liberdade, e Sig significa nível de significância). ${ }^{*} p<.05$ 


\section{4 - Conclusões}

Os resultados obtidos mostram que ambos os grupos (Experimental e Controlo) obtiveram ganhos a nível da Leitura/Execução Musical e Reconhecimento Auditivo. Apesar de se terem verificado ganhos longitudinais no Grupo de Controlo, o desempenho elevado alcançado pelo Grupo Experimental no pós-teste em todas as características, permite retirar fortes indicadores que as atividades pedagógico-musicais desenvolvidas no seio do MoMEA, construídas numa linha conceptual que congrega fundamentos basilares da Teoria e Aprendizagem Musical de GORDON (2000) e princípios do Modelo de Improvisação de AZZARA (2008), produziram efeitos no desempenho musical dos participantes. Esta afirmação encontra sustentação e fundamentação nas condições experimentais similares e design de aplicabilidade do modelo. Contudo, o facto de o impacto da instrução se ter verificado estatisticamente significativo apenas para algumas das características permite-nos colocar algumas questões secundárias para reflexão:

1. Por que é que os efeitos da instrução não foram sentidos de uma forma significativa em algumas características?

2. Por que não existiu qualquer evolução no âmbito do reconhecimento dos Elementos Expressivos e Diversidade Estilista e Instrumental?

3. Por que razão na variável Solfejo o processo de instrução não produziu nenhuma evolução no Grupo Experimental, ao contrário do que foi verificado no Grupo de Controlo?

Apesar de todas estas questões possam ser alvo de desenvolvimento através de procedimentos experimentais, podemos desde já avançar algumas hipóteses a partir da inferência dos dados empíricos e de outros contextos teóricos subjacentes. Assim, no caso da primeira questão um dos aspetos que possa estar na base dos resultados obtidos é o próprio processo sequencial utilizado. Ou seja, como todo o desenvolvimento do MoMEA é apoiado nas linhas conceptuais de Gordon (2000) e AZZARA (2008), orientadas por uma coesão progressiva de ciclos e estádios (generalização e inferência), leva que o processo seja moroso em toda a sua amplitude. A este fator, acrescenta-se todo o imput de informação e construção musical dos participantes deste estudo obtido durante todo o percurso académico e artístico, onde os processos de ensino e aprendizagem são caracterizados por tornar e considerar os elementos musicais como significativos em si mesmos.

Em relação à segunda questão, a hipótese a considerada poderá ter na sua base o modelo metodológico proposto por KARPINSKI (2000). Ou seja, a análise dos dados mostra que a diferença evolutiva nestas variáveis não é reveladora de diferenças significativas. Este facto, pode ser explicado pelo motivo destes elementos representarem recursos básicos na aquisição de competências auditivas (KARPINSKI, 2000). Esta inferência vai de encontro às ilações de LEVITIN (2011), que avigora a ideia da facilidade com que a maioria das pessoas consegue identificar os sons como reconhecem uma cor, facto que se deve unicamente pela identificação do timbre e não pela altura. Neste particular, SCHOENBERG $(1999$, p.578) refere que “... o som se faz percetível através do timbre, do qual a altura é uma dimensão...". 
No que concerne à terceira questão, os valores atingidos eram de alguma forma esperados por nós, uma vez que o processo de ensino e aprendizagem relativo ao Grupo de Controlo, apresenta em toda a sua linha de instrução, o objetivo primo de uma valorização das práticas de leitura e escrita da notação musical. Devemos salientar que a simples capacidade de nomear e identificar simbologia musical não representa uma manifestação de literacia musical ou mesmo uma preparação para tal (GORDON, 2000). Esta problemática da leitura e escrita notacional mereceu desde sempre a atenção e é motivo de alguma celeuma no universo do Ensino da Música. Estabelecendo um comparativo com a literacia linguística, facilmente podemos constatar que a construção de uma literacia musical não se deve remeter unicamente à leitura e escrita, mas deverá também incluir a escuta e a execução musical. A leitura e a escrita deverão ser meio e não representar o fim. De uma forma geral, os resultados alcançados permitem vislumbrar a possibilidade de existir uma aplicabilidade do Modelo Multidimensional de Educação Auditiva (MoMEA) numa escala mais abrangente. Contudo, dado o processo sequencial de ensino e aprendizagem, serão necessárias práticas e experiências de alguma continuidade e regularidade para que todas as competências possam ser alcançadas.

\section{Referências}

1. AZZARA, C. (1993). The effect of audiation based improvisation techniques and elementary instrumental student's music achievement. Journal of Research in Music Education. n.4, v.41, p.328-342.

2. (2008). Improvisation and choral musicianship. In: The school choral program: Philosophy, planning, organizing, and teaching. Ed. por Holt e Jordan, Chicago: GIA. Publications, Inc.

3. AZZARA, C.; GRUNOW, R. (2006). Developing musicianship through improvisation. Chicago: GIA Publications, Inc.

4. BARBOSA, M. (2009). Percepção musical como compreensão da obra musical: contributos a partir da pespectiva histórica-cultural (Tese de Doutoramento não publicada). Faculdade de Educação da Universidade de São Paulo, Brasil.

5. BERNARDES, V. (2001). A percepção musical sob a ótica da linguagem. Revista da ABEM. v.6, p.73-85.

6. BISQUERRA, R.; SARRIERA, J. C.; MATÍNEZ, F. (2007). Introdução à estatística: enfoque informático com o pacote estatístico SPSS. Porto Alegre: Artmed.

7. BLUESTINE, E. (1995). The ways children learn music. Chicago: GIA Publications.

8. BOLTON, B.; TAGGART, C.; REYNOLDS, A.; VALERIO, W.; GORDON, E. (2001). Jump right in: The music curriculum, book 2 (Rev. ed.). Chicago: GIA.

9. CARDOSO, M. (2015). A (des)construção (Tese de Doutoramento). Departamento de Educação e Psicologia da Universidade de Trás-os-Montes e Alto Douro, Portugal.

10. CASPURRO, M. (2006). Efeitos da aprendizagem da audição da sintaxe harmónica no desenvolvimento da improvisação (Tese de Doutoramento). Departamento de Comunicação e Arte da Universidade de Aveiro, Portugal.

11. CRUZ, C. (2013). 0 tradicional e o erudito, o som e a escrita, a técnica e a arte. Uma Choradinha açoriana e uma Ungaresca italiana, com Saltarello. Revista de Educação Musical. v.139, p.22-30.

12. DAHLHAUS, C. (1977). Estética musical. Lisboa: Edições 70. 
CARDOSO, M.; MORGADO, E.; SILVA, L. (2018) Os efeitos de uma proposta didática de Educação Auditiva no desempenho musical. Per Musi. Belo Horioznte: UFMG. p.1-18.

13. FISKE, H. (1995). Investigação experimental. In: Introdução à Investigação em Educação. Ed. por Kemp, Lisboa: Fundação Calouste Gulbenkian.

14. FORTIN, M. (1999). 0 processo de investigação: da concepção à realização. Loures: Lusociência.

15. FREIXO, M. (2011). Metodologia Científica: Fundamentos, Métodos e Técnicas (3. ${ }^{\text {a }}$ E.). Lisboa: Instituto Piaget

16. GORDON, E. (2002). Rating scales and their uses for measuring and evaluating achievement in music performance. Chicago: GIA Publications.

17. (2009). Rhythm: Contrasting the implications of audiation and notation. Chicago: GIA Publications.

18. GREEN, L. (2008). Music, informal learning and the school: a new classroom pedagogy. Farnham-Surrey: Asghate.

19. KARPINSKI, G. (2000). Aural skills acquisition: The development of listening, reading, and performing skills in college-level musicians. Oxford: Oxford University Press.

20. KNOWELS, M.; HOLTON, E.; SWANSON, A. (2005). Adult Learner. Boston: Elsevier Butterworth Heinemann.

21. LANGE, D. (2005). Together in Harmony: Combining Orff Schulwerk and Music Learning Theory. Chicago: GIA Publications.

22. LEVITIN, D. (2011). This is your brain on music: Understanding a human obsession. Atlantic Books Ltd.

23. . (2013). Uma Paixão Humana: 0 seu Cérebro e a Música. Lisboa: Editorial Bizâncio.

24. MCCLARY, S. (1991). Feminine Endings. Music, Gender, and Sexuality. Minnesota.

25. OTUTUMI, C. (2008). Percepção musical: situação actual da disciplina nos cursos superiores de música. Campinas: Instituto das Artes.

26. RODRIGUES, H. (1998). Pequena Crónica sobre Notas de Rodapé na Educação Musical. Reflexões a propósito da teoria da aprendizagem musical. Boletin da APEM. v.99, p.15-25.

27. ROVAI, A.; BAKER, J.; PONTON, M. (2013). Social Science Research Design and Statistics: A Practitioner's Guide to Research Methods and IBM SPSS. Chesapeake: Watertree Press.

28. SARAMAGO, J. (2008). A viagem do elefante. Caminho.

29. SCHONBERG, A. (1999). Harmonia. São Paulo: Editora UNESP.

30. SISCAR, M. (2005). O Coração Transtornado. In: Jacques Derrida: pensar a desconstrução. Org. por Nascimento, São Paulo: Estação Liberdade.

31. TAGGART, C.; BOLTON, B.; REYNOLDS, A.; VALERIO, W.; GORDON, E. (2006). Jump right in: The music curriculum (Livro IV). Chicago: GIA.

32. Trochim, W. (2000). The Research Methods Knowledge Base (2 $\stackrel{a}{a}$ ed.). Cincinnati: Atomic Dog Publishing. 33. TUCKMAN, B. (2005). Manual de Investigação em Educação (3ª ed.). Lisboa: Fundação Calouste Gulbenkian. 34. VARGAS, A. (2013). Como se ouve aquilo que não se conhece. Revista de Educação Musical. v.139, p.13-21. 
CARDOSO, M.; MORGADO, E.; SILVA, L. (2018) Os efeitos de uma proposta didática de Educação Auditiva no desempenho musical. Per Musi. Belo Horioznte: UFMG. p.1-18.

35. WEINBERG, S. L.; ABRAMOWITZ, S. K. (2008). Statistics using SPSS: An integrative approach. Cambridge University Press.

36. YUNUS, F. (2010). Statistics Using SPSS: An Integrative Approach. Journal of Applied Statistics. n.12, v.37, p.2119-2120.

Nota sobre os autores

Mário Cardoso é Professor Adjunto do Departamento de Educação Musical da Escola Superior de Educação do Instituto Politécnico de Bragança, Portugal. É Presidente da Comissão Científica e Director de Curso do Mestrado em Ensino de Educação Musical no Ensino Básico da Escola Superior de Educação do Instituto Politécnico de Bragança. Faz parte da Coordenação (Coordenador-Ajunto) do Departamento de Educação Musical e membro do Centro de Investigação em Educação Básica na mesma instituição. As áreas de interesse e atividade científica estão centradas nas Ciências Musicais (interpretação, análise e composição musical), Estudos Artísticos (arte e tecnologia, estudos musicais, estudos fílmicos e da imagem) e Ciências da Educação (ensino e psicologia da Música). Atualmente é investigador de Pós-Doutoramento na Universidade de Trás-os-Montes e Alto Douro. ORCID - 0000-0003-3645-9641

Elsa Morgado é Doutora em Ciências da Educação pela Universidade de Trás-os-Montes e Alto Douro; Mestre em Biologia e Geologia (para o ensino); Especializada em Educação Especial (domínio Cognitivo e Motor) e Licenciada em Biologia e Geologia (ensino de). Investigadora Auxiliar Convidada do Centro de Estudos Filosóficos e Humanísticos da Universidade Católica Portuguesa - Braga; Investigadora de pós-Doutoramento na Universidade de Trás-os-Montes e Alto Douro. Tem desenvolvido uma regular atividade e investigativa no domínio das Ciências da Educação; Educação Especial; Supervisão Pedagógica e Estágio de Inserção Profissional. ORCID - 0000-0002-3653-7876

Levi Silva é Mestre e Doutor em Educação pela Universidade de Salamanca. Professor Auxiliar da Escola das Ciências Humanas e Sociais da Universidade de Trás-os-Montes e Alto Douro. Desenvolveu estudos de Pós-doutoramento na Universidade de Coimbra (2009-11) e na Universidade de Santiago de Compostela (2007). Membro do CITAR (Centro de Investigação em Ciência e Tecnologias da Artes da Universidade Católica Portuguesa. Diretor da Revista Europeia de Estudos Artísticos (European Review of Artistic Studies). Tem desenvolvido uma regular atividade investigativa no domínio da Artes e Educação com especial enfoque na criação / direção artística e na orientação de trabalhos de investigação avançada (mestrado, doutoramento e pós-doutoramento). ORCID - 0000-0001-6603-034X 